\title{
SPECIAL FEATURES OF PEROXY RADICALS ON
} PLASMA-IRRADIATED POLYMERS

\author{
MASAYUKI KUZUYA,* MASAMI SUGITO AND SHIN-ICHI KONDO \\ Laboratory of Pharmaceutical Physical Chemistry, Gifu Pharmaceutical \\ University, 5-6-1, Mitahora-Higashi, Gifu 502, JAPAN
}

One of the characteristics of plasma irradiation is the effective energy transfer to solid surface to create a large amount of stable free radicals on a variety of polymers.[1] Plasma-induced surface radicals thus formed permit reactions for surface modifications in several different ways (plasma treatment).[2] Studies of auto-oxidation process of plasma-irradiated polymers by air exposure are of practical importance for the industrial application, because such materials are used in atmosphere. In the auto-oxidation process, it is of special importance to clarify the peroxy radical formation (ROO) and its structural relaxation. Peroxy radicals in polymers formed mostly by high energy radiation have been extensively studied by electron spin resonance (ESR).[3] The temperature-dependent ESR spectra of peroxy radicals derived from the mid-chain alkyl radicals of several polymers have recently been used as spin probes to study the molecular motion of polymer chains.[4-6] Yet, the detailed ESR studies of peroxy radicals derived from plasma-irradiated polymers have not been reported.

We report the special features of the peroxy radicals formed from plasma-irradiated acrylic resins such as polymethacrylic acid (PMAA) and its methyl ester (PMMA), and in two kinds of copolymers of MMA and MAA, commercially known as Eudragit L100 (6:4) and Eudragit S100 (7:3), based on the ESR including the time- and temperature-dependent spectra.

Powdered acrylic resins $(50 \mathrm{mg})$ were placed in a specially-designed ampule $(30 \mathrm{~mm}$ i.d., $100 \mathrm{~mm}$ long ) connected with capillary tube at the uppermost part of the ampule, and Ar plasma irradiation was carried out for a prescribed period of time according to the method as reported earlier.[1]

We have already shown that the observed ESR spectra were deconvoluted with the aid of the systematic computer simulations invoking four kinds of isotropic compo- 
nent spectra, nine-line spectrum (I), doublet (II), seven-line spectrum (III) and broad single-line spectrum (IV). These component spectra were assigned to the terminating radical (1), $\sim \mathrm{CH}_{2}-\dot{\mathrm{C}}(\mathrm{COOR})\left(\mathrm{CH}_{3}\right)$, mid-chain alkyl radical $(2), \sim(\mathrm{COOR})\left(\mathrm{CH}_{3}\right) \mathrm{C}-\dot{\mathrm{C}} \mathrm{H}-\mathrm{C}\left(\mathrm{CH}_{3}\right)(\mathrm{COOR}) \sim$, monomer-derived radical (3), $\left(\mathrm{CH}_{3}\right)_{2} \dot{\mathrm{C}}(\mathrm{COOR})$ and immobilized dangling bond sites (4), respectively.

The formation of peroxy radicals was furnished by opening the ampule and exposing the above-mentioned radicals in dry atmosphere at room temperature. In fact, it was shown that plasma-induced radicals of acrylic resins, when in contact with oxygen, were rapidly converted to the corresponding peroxy radicals in all cases. The formation of peroxy radicals was ca. $70 \%$ in yield based on the comparison of the spectral intensities between the plasma-induced radicals and the peroxy radicals, indicating that some of the resulting peroxy radicals are very unstable at room temperature to escape ESR detection.

The typical time-dependent ESR spectra of the peroxy radicals formed from plasma-irradiated Eudragit L100 are shown in Fig. 1, as a representative example. The similar spectra were also observed in the other three acrylic resins under the same treatments.

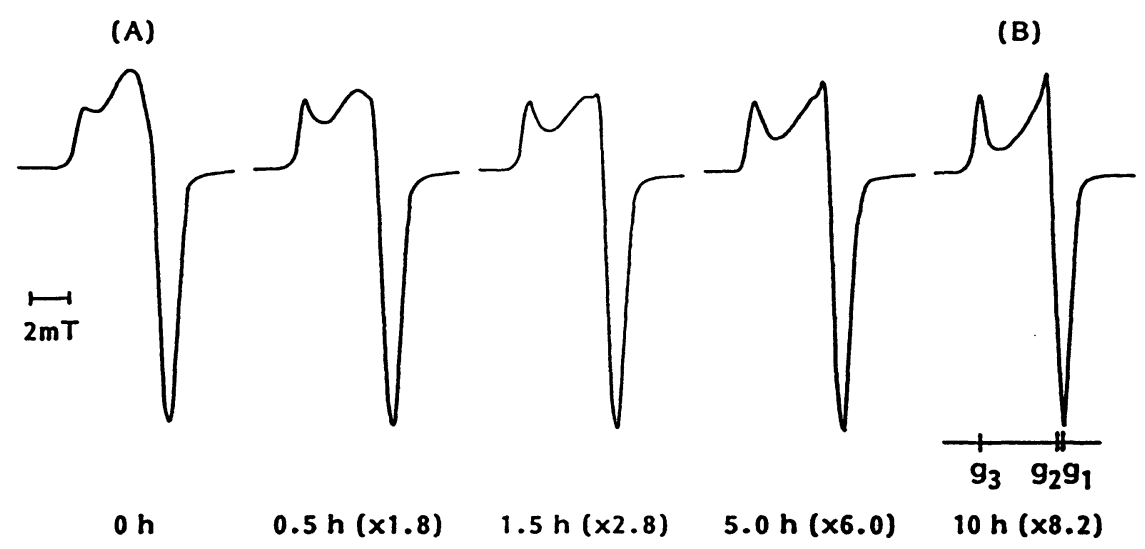

Fig. 1 Time-dependent ESR spectra of peroxy radicals at room temperature derived from plasma-irradiated Eudragit L100. The principal g-values $\left(\mathrm{g}_{1}=2.0051, \mathrm{~g}_{2}=2.0068\right.$, $\mathrm{g}_{3}=2.0345$ ) of the spectrum (B) were estimated by the simulated spectrum.

It is seen that the spectrum (A) measured immediately after oxygen exposure is largely different in shape from the spectrum (B) measured when stored for $10 \mathrm{~h}$ at room temperature. The spectrum (B) is a well-known asymmetric spectrum for a peroxy radical due to the g-anisotropy, and remained unchanged in shape for a long period of time. This type of asymmetric spectrum (B) has never been shown previously at room temperature, but has only been observed at very low temperature in many other systems.[3] It can be considered that 
many trapping sites of the peroxy radicals exist due to different environments in polymers, hence the motional character of each of the trapping site is different.[3] In this sence, the ESR spectrum of peroxy radicals formed should be a superposition of many kinds of spectral patterns with different sets of principal g-values.

Thus, it appears that the spectrum (A) in Fig. 1 is a mixture of peroxy radicals with various kinds of molecular motions, and less stable radicals decay fast, preserving the stable radicals with more discrete molecular motions. This accounts for the spectral decay as well as the spectral change shown in Fig. 1.

The temperature-dependence of the spectra (A) and (B) are shown in Fig. 2. The temperature-dependence of the spectrum $(A)$ is apparently similar to the time-dependent spectra shown in Fig. 1, and varies reversibly in shape over a wide range of temperature, while the spectrum (B) remained nearly unchanged except for the increment of the spectral intensities with the spectral narrowing at lower temperatures due to the changes of Boltzmann distribution. The temperature-dependent spectra of peroxy radicals similar to the spectra (A) are known in many other systems.[3]

(A)

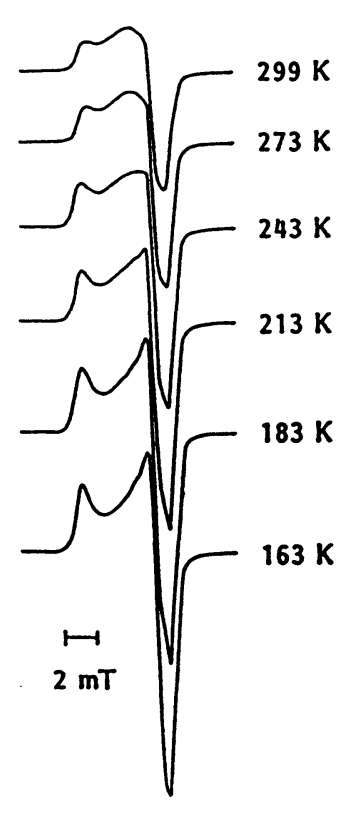

(B)

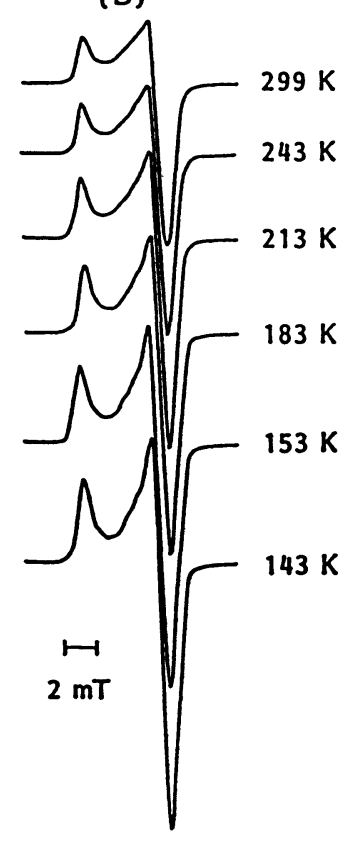

Fig. 2 Temperature-dependent ESR spectra of peroxy radicals of spectra (A) and (B) shown in Fig. 1.

Since plasma irradiation produces more than one surface radicals, the observed ESR spectra of peroxy radicals are composed of several component spectra. It was found, however, that 
the nature of peroxy radicals should be analyzed, according not to the structures of plasma-induced radicals, but to the motional character of peroxy radicals on a different environment of the trapping sites of polymer surfaces, each of which would have an unique amount of g-anisotropy. The results derived from such analyses will be reported in a forthcoming paper.

Acknowledgments This work was supported in part by a Scientific Research Grant from the Ministry of Education, Science, Sports and Culture of Japan (Grant No. 07672319), which is gratefully acknowledged.

\section{References}

1. a) Kuzuya M., Noguchi A., Ishikawa M., Koide A., Sawada K, Ito A., Noda N., J. Phys. Chem., 95, 2398 (1991). b) Kuzuya M., Noguchi A., Ito H., Kondo S., Noda N., J. Polym. Sci., Polym. Chem., 29, 1 (1991). c) Kuzuya M., Ishikawa M., Noguchi A., Ito H., Kamiya K., Kawaguti T., J. Mater. Chem., 1,387 (1991). d) Kuzuya M., Kamiya K., Kawaguchi T., Proc. Jpn. Symp. Plasma. Chem., 4,317 (1991). e) Kuzuya M., Ito H., Kondo S., Noda N., Noguchi A., Macromolecules, 24, 6612 (1991). f) Kuzuya M., Noda N., Kondo S., Washino K, Noguchi A., J. Am. Chem. Soc., 114, 6505 (1992). g) Kuzuya M., Kondo S., Ito H., Noguchi A., Appl. Surf. Sci., 60/61, 416 (1992). h) Kuzuya M., Ishikawa M., Noguchi A., Sawada K., Kondo S., J. Polym. Sci. Polym. Chem., 30,379 (1992).

i) Kuzuya M., J. Photopolym. Sci. Tech., 5, 407 (1992).(Review) j) Kuzuya M., Sawada K., Takai T., Noguchi A., Polymer J., 25, 75 (1993). k) Kuzuya M., Kamiya K. Yanagihara Y., Matsuno Y., Plasma Sources Sci. Technol., 2, 51 (1993). 1) Kuzuya M., Niwa J., Ito H., Macromolecules, 26, 1990 (1993). m) Kuzuya M., Morisaki K, Niwa J., Yamauchi Y., Xu K, J. Phys. Chem., 98, 11301 (1994). n) Niwa J., Kuzuya M., Proc. Jpn. Symp. Plasma. Chem., 7, 59 (1994). o) Kuzuya M., Niwa J., Noguchi T., Polymer J., 27, 251 (1995). p) Kuzuya M., Yamauchi Y., Niwa J., Kondo S., Sakai Y., Chem. Pharm. Bull., 43, 2037 (1995).

2. M. Hudis, Techniques and Applications of Plasma Chemistry, ed. by J. R. Hollahan, A. T. Bell, John Wiley, New York, 1974.

3. Kashiwabara H., Shimada S., Hori Y., Sakaguchi M., Ad. Polym. Sci., 82, 164 (1987). and references cited therein.

4. Schlick S., Kevan L., J. Phys. Chem., 83,3424 (1979).

5. Suryanarayana D., Kevan L., J. Phys. Chem., 86, 2042 (1982).

6. Suryanarayana D., Kevan L, Schlick S., J. Am. Chem. Soc., 104, 668 (1982). 